\title{
Pervasive Computing for Automobiles: An Approach to Maximize User Convenience and Safety Using VANETs
}

\author{
Sini Shibu ${ }^{1}$ and Sanjeev Jain ${ }^{2}$
}

\begin{abstract}
Invention of wheel was perhaps the most significant invention in the evolution of mankind. From the earliest carts to the present day automobiles, industry has witnessed dramatic changes and innovations. But even today, automobiles are equipped with mechanical, electrical and electronic devices that are not smart enough to take decisions on behalf of the user. The way mobile computing devices and applications are developed, deployed and used in automobiles today falls short of the potential for pervasive computing. This paper attempts to build a model of an automobile that uses pervasive computing to maximize user convenience and safety. The concept used is that of Vehicular Ad-hoc Networks (VANETs). The major concern in a VANET is to provide reliable and efficient packet delivery mechanism taking into consideration the limited battery back-up of the mobile devices and also the available bandwidth. Moreover, data communication between vehicular mobile devices can minimize head-on collisions by proper auto-break mechanism implementation using Ultra Sound Generators and sensors. The implementation issues are also addressed in this paper. Ultimately, this paper presents ideas that could make driving simpler and safer as compared to the present day.
\end{abstract}

Index Terms-Pervasive Computing, Wireless Sensor Networks (WSN), Mobile Computing, Vehicular Ad hoc Networks (VANETs), User System Interaction (USI), Sonar Vision, Ultra Sound Generator(USG)

\section{INTRODUCTION}

\section{A. Pervasive Computing}

In the early 90s Mark Weiser introduced the concept of Ubiquitous Computing which is now better known as Pervasive Computing [8]. This concept is considered to be the third wave of computing technologies to emerge since computers first appeared:

- First Wave was the Mainframe computing era where one computer was shared by many people, via workstations.

- Second Wave was the Personal computing era in which one computer was used by one person and it required a conscious interaction between the user and the system (usually a desktop or a laptop).

- Third Wave is the Pervasive computing era in which there might be one user and many computers to aid him in the

This work was supported in part by the Department of Computer Science and Engineering, Lakshmi Narain College of Technology (LNCT), Bhopal, MP, India. Technical support was given by the lab co-ordinators of Department of Computer Science and Engineering, Lakshmi Narain College of Technology (LNCT) and Technocrats Institute of Technology, Bhopal, for performing computation and sensor based experiments for this work.

Sini Shibu and Sanjeev Jain are with the Department Computer Science and Engineering Lakshmi Narain College of Technology Bhopal, India background. Millions of computers would be embedded in the environment, allowing technology to recede into the background. Deliberate user system interaction will also be minimized with the use of pervasive systems.

With the emergence of Pervasive computing era and the developments in networking and hardware a lot of things have become realizable in the recent past. This has lead to a wider interest in pervasive computing in the Computer Science research community. The focus today is on the imminent transformation of computing from a desktop technology as we know it now to a pervasive "aware" technology where computers, ranging from supercomputers to desktops to embedded, are active but invisible partners in enhancing our quality of life in all its arenas[10].

\section{B. Wireless Sensor Networks}

A wireless sensor network (WSN) is a wireless network of independent devices with sensors which may be distributed over a geographical area to cooperatively monitor physical or environmental conditions, such as temperature, sound, vibration, pressure, motion or pollutants, etc.[4]. Today, wireless sensor networks are used in many industrial and civilian application areas, such as industrial process monitoring and control, machine health monitoring, environment and habitat monitoring, healthcare applications, home automation, and traffic control. Every node in a sensor network has one or many sensors along with either a wireless communication device or a radio transceiver connected to a microcontroller and a battery. The size of a sensor node may vary from the size of a computer to microscopic sensor based devices. The cost of sensor nodes is similarly variable, ranging from hundreds of dollars to a few cents, depending on the size of the sensor network and the complexity required of individual sensor nodes. The cost of the sensor node would depend upon the bandwidth of data transmission, computational speed and real time application support provided by the node. A sensor network normally constitutes a wireless ad-hoc network, meaning that each sensor supports a multi-hop routing algorithm (several nodes may forward data packets to the base station). Wireless Sensor Networks are going to replace all static networks in the times to come because mobility and wireless computing is the need of the hour. In automobiles, WSN can be implemented for making driving more safer and to provide a pervasive environment for vehicles.

\section{Vehicular Ad-hoc Networks}

Vehicular Ad-Hoc Network, or VANET, is a form of Mobile ad-hoc network, to provide communications among nearby vehicles and between vehicles and nearby fixed 
equipment, usually described as roadside equipment. The main goal of VANET is providing safety and comfort for passengers[12]. To this end a special electronic device will be placed inside each vehicle which will provide Ad-Hoc Network connectivity for the passengers. This network tends to operate without any infra-structure or legacy client and server communication. Each vehicle equipped with VANET device will be a node in the Ad-Hoc network and can receive and relay others messages through the wireless network. Collision warning, road sign alarms and in-place traffic view will give the driver essential tools to decide the best path along the way. There are also multimedia and internet connectivity facilities for passengers, all provided within the wireless coverage of each car. Automatic payment for parking lots and toll collection are other examples of possibilities inside VANET. Most of the concerns of interest to MANETs are of interest in VANETs, but the details differ. Rather than moving at random, vehicles tend to move in an organized fashion. The interactions with roadside equipment can likewise be characterized fairly accurately. And finally, most vehicles are restricted in their range of motion, for example by being constrained to follow a paved highway.

\section{Ultrasound Generator (USG)}

Ultrasound is cyclic sound pressure with a frequency greater than the upper limit of human hearing. Although this limit varies from person to person, it is approximately 20 kilohertz $(20,000$ hertz) in healthy, young adults and thus, 20 $\mathrm{kHz}$ serves as a useful lower limit in describing ultrasound. The production of ultrasound is used in many different fields, typically to penetrate a medium and measure the reflection signature or supply focused energy. An ultrasound generator is an electronic machine that can generate ultra sound waves.

The rest of the paper is organized as follows: Section II explains the need for safer driving mechanisms, Section III enumerates the existing technologies for safe automobiles, Section IV presents the proposed automobile model, Section $\mathrm{V}$ presents the limitations of the proposed model, Section VI concludes the paper.

\section{NEED FOR SAFER DRIVING MECHANISM}

In the industrialized countries, and also in many developing countries, on an average one hospital bed in ten is occupied by an accident victim. Traffic accidents are a major cause of severe injuries in most countries. Developing countries have nearly four times the number of deaths from these causes as the developed world. The 1999 WHO publication "Injury: A Leading Cause of the Global Burden of Disease," reports that the leading injury-related cause of death among people aged 15-44 years is traffic injuries[1]. Hence, the major concern today still remains to make automobiles safer and easier to be handled. Integrating pervasive systems with present day automobile technology can not only prevent accidents but also make driving easier. In their work Luis E. Palafox and J. Antonio Garc'1a-Macias [3] have created a model to capture voice in a ubiquitous home environment. This voice capture mechanism is the major motivation of this work and in this paper we have proposed the idea of capturing ultra sound for ubiquitous automobile applications.

\section{Existing Technologies for SAFE Automobiles}

- $\quad$ Electronic stability control (ESC):

One of the best features of Mercedes S class is Electronic stability control (ESC) which is a computerized technology that improves the safety of a vehicle's handling by detecting and preventing skids. When ESC detects loss of steering control, ESC automatically applies individual brakes to help "steer" the vehicle where the driver wants to go. Braking is automatically applied to individual wheels, such as the outer front wheel to counter oversteer, or the inner rear wheel to counter understeer. Some ESC systems also reduce engine power until control is regained.

\section{- Sonar Vision:}

Sonar Vision is an anti-collision device designed to prevent drivers from backing into objects. Sonar Vision has been designed to be inconspicuous and satisfies even the most discerning vehicle owner's aesthetic sense. This innovative electronic device offers that additional control for any driver and protection for everything and everybody else, making everyday living easier and safer[2].

\section{Proposed Automobile Model}

As stated in section II, since there is an ever growing need for safer driving mechanism, this paper attempts to project a model of a safe vehicle.

\section{A. USG based collision avoidance mechanism}

In the proposed model, every vehicle would be equipped with a device known as a VANET node. A VANET node is a computing device loaded with an operating system and utilities as in a typical laptop. It would be mounted on the front side of an automobile so that the driver could operate it easily. It essentially comprises of a face recognition software and a camera mounted just towards the top of the wind shield in front of the driver. Additionally the VANET node is connected to an Ultra Sound Generator (USG) and a sensor. The USG and sensor is mounted centrally in the vehicle. The USG would be emitting a sound wave of wavelength $\lambda$ that would terminate at distance $d$. The sound waves emitted by the USG will be continuously monitored by the sensor. The sensor would further provide input to the distance intensity graph generator and a software would continuously monitor the graph being generated at every instance of time for a rotation of $360^{\circ}$. The entire mechanism is explained below:

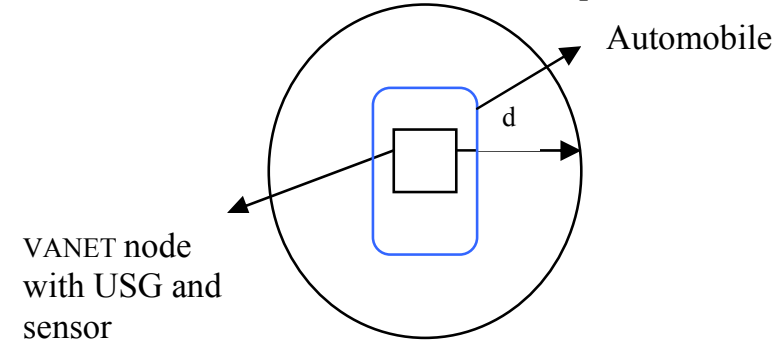

Figure 1 Automobile with VANET node

The safety distance $\mathrm{d}$ would be set in the VANET node depending upon the dimensions of the automobile. When the USG generates a sound wave its intensity would be decreasingly maintained by the VANET node which is depicted below: 


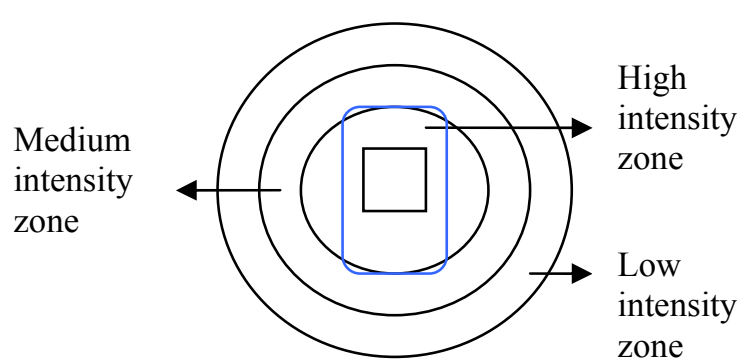

Figure 2 Intensity zones of an automobile

In figure $3, \mathrm{~d}$ is the safety distance and $\mathrm{d}_{\mathrm{th}}$ is the threshold distance. Graphically, the intensity zones of a VANET node could be represented for a rotation of $360^{\circ}$ as shown below:

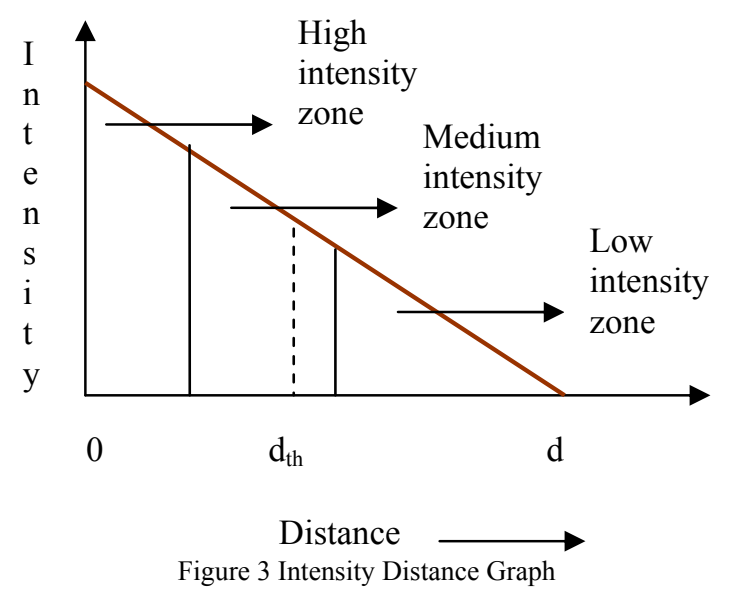

When two automobiles, each equipped with a VANET node, come in close proximity to each other there would be interference between the intensity zones of the two vehicles. In the proposed model a continuous observation of the distance intensity graph is done by a software and appropriate brake mechanism would be activated in case the vehicles come very close to each other. Consider two VANET nodes equipped automobiles $\mathrm{A}$ and $\mathrm{B}$ coming nearer to each other head on moving in the direction as indicated by the arrows. The interference in their intensity zones could be depicted as:

Interference between intensity zones

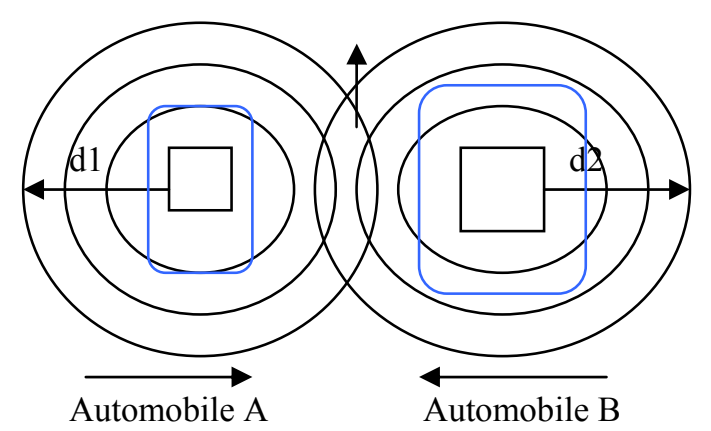

Figure 4 Intensity interference between two vehicles

This interference would result in a non decreasing graph since the intensities of both the sound waves overlap each other. Through a 360o rotation of VANET node A sensor, the distance-intensity graph of Automobile A for an instance time $t$ would deviate from the original graph as shown below:

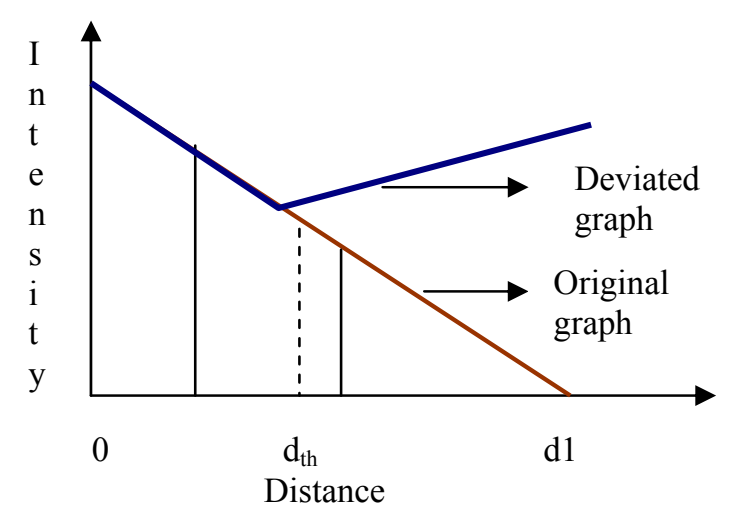

Figure 5 Deviation in Intensity Distance Graph for Vehicle A

The graphs generated could be fed as input to a monitoring mechanism that calculates a vector as follows:

where

$$
\mathrm{V}=\left[\begin{array}{llll}
\mathrm{A}_{1} & \mathrm{~A}_{2} & \mathrm{~A}_{3} & \ldots . . \mathrm{A}_{\mathrm{n}}
\end{array}\right]
$$

$\mathrm{n}$ is the number of intensity distance graph observation samples taken during a $360^{\circ}$ rotation sensing

$A_{n}\left\{\begin{array}{l}1 \text { if graph structure is non-decreasing prior to } d_{\text {th }} \\ 0, \text { otherwise }\end{array}\right.$

The vectors could be generated at greater frequencies and the values could be compared. Suppose the value of $n$ is taken as 6. This would imply that values of vector $\mathrm{V}$ will be calculated at say $0^{\circ}, 60^{\circ}, 120^{\circ}, 180^{\circ}, 240^{\circ}$ and $300^{\circ}$ for the first iteration. For the second iteration they could be varied by say $5^{\circ}$. Thus, vectors could be generated and stored in a FIFO buffer for comparison. While comparison, if $1 \mathrm{~s}$ are generated in the vector successively in each iteration then the braking mechanism of the automobile needs to be activated. By increasing the value of $\mathrm{n}$, accuracy could be achieved but at the cost of computational complexity and time consumption. If the sensor mechanism of VANET node A detects a graph structure that is non-decreasing in nature at any instant $t$ during its $360^{\circ}$ rotation sensing, then the auto brake mechanism would be activated so as to avoid head on collision. Also it should monitor that the deviation occurs within the threshold distance $\mathrm{d}_{\mathrm{th}}$. Another consideration could be to start the activation process of the auto braking mechanism at the slightest interference and to completely apply brakes when the interference crosses the threshold distance $d_{\text {th }}$. The same mechanism will also take place in Automobile B thus avoiding a head-on collision between two approaching vehicles The brake mechanism should be activated within a very small time span to avoid collisions. This frequency will depend upon the speed of the sensing mechanism and vector generation and comparison. For automobiles, it would be preferable to keep the value of $n$ between 6 and 10 to achieve accuracy with minimum delay. This mechanism could be summarized in the following flowchart: 


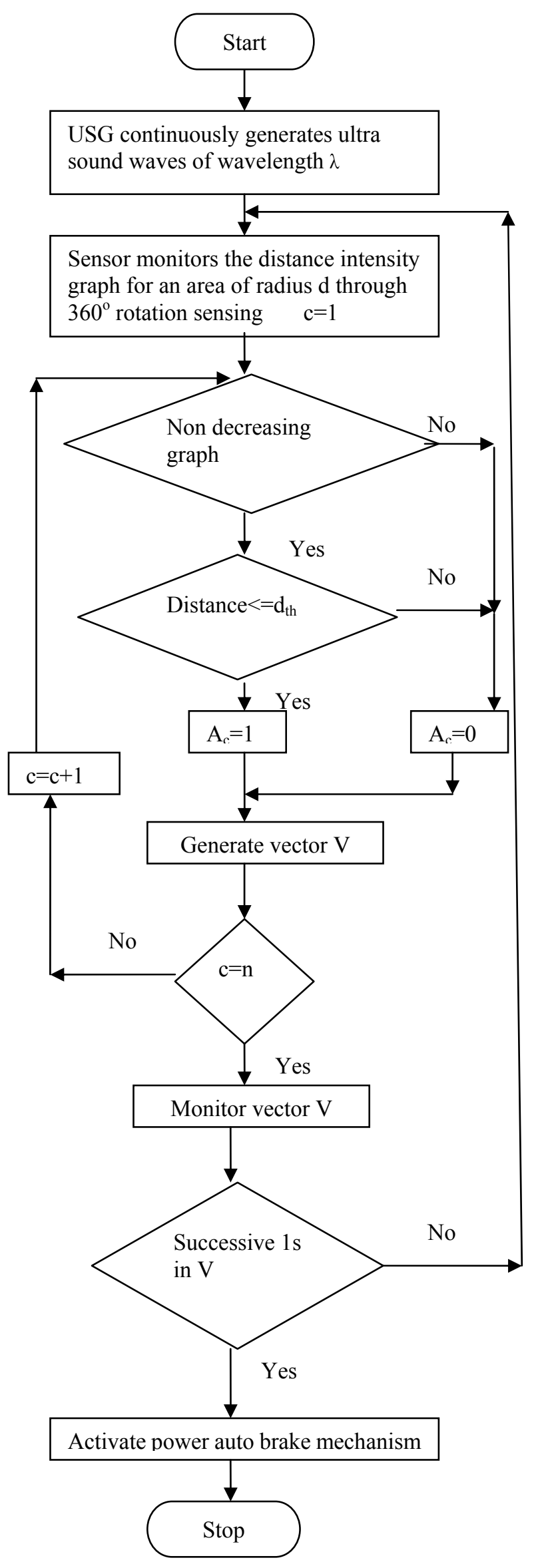

collisions, certain considerations need to be addressed or else it may lead to serious traffic hazards.

\section{Consideration 1:}

According to this model, the ultra sound waves should be propagated throughout the area of radius $d$ of a VANET node. But this may cause problems to overtaking vehicles. Consider the VANET node shown below:

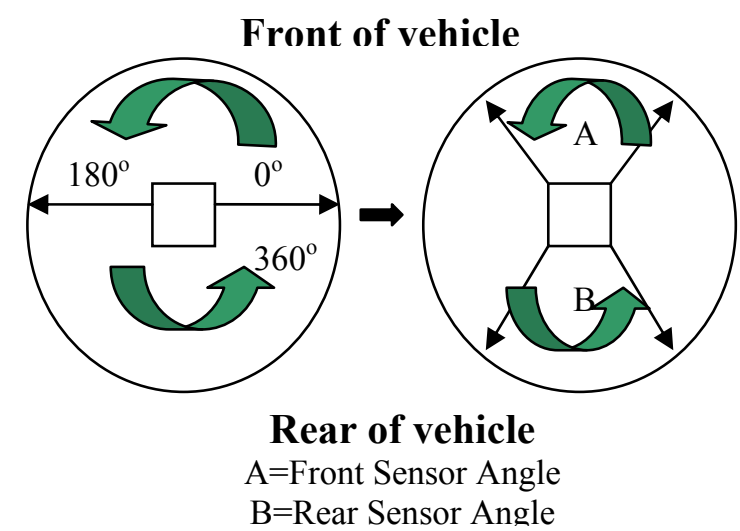

Figure 7 Sensor Angles

If two vehicles with VANET nodes are moving in the same direction parallel to each other, they would surely cause intensity interference and the vehicles would be stopped. Hence, the auto brake mechanism should be activated only in case when the interference of the intensity zones would occur towards the front and the rear side of the vehicle or else overtaking of vehicles will not be possible. This could be done by setting the sensor angle of the VANET node. Thus, typical front sensor angle values may vary from $45^{\circ}$ to $135^{\circ}$ and rear sensor angle value would lie between $225^{\circ}$ to $315^{\circ}$ depending upon the individual vehicle settings. The sensor would only take action if the intensity interference occurs in this range.

\section{Consideration 2:}

In the proposed model, if the sensor detects intensity interference it would activate the auto brake mechanism. This could be troublesome if auto brakes are applied even at the slightest interference. If the interference is only in the low intensity zone, the auto brake mechanism should not be activated. Thus, a threshold value of distance $d$ should be initialized in the VANET node so that if the intensity distance graph deviates within the threshold distance $\mathrm{d}_{\mathrm{th}}$ only then the auto brake mechanism should be activated.

\section{Consideration 3:}

The proposed model would help in avoiding head on and rear collisions, but if an automobile approaches another perpendicularly, then only one of the vehicles would be stopped. Consider the following scenario where two vehicles are moving in the direction as shown by the arrows:

Figure 6 Flowchart of USG-Sensor mechanism

Although this mechanism would help in avoiding 

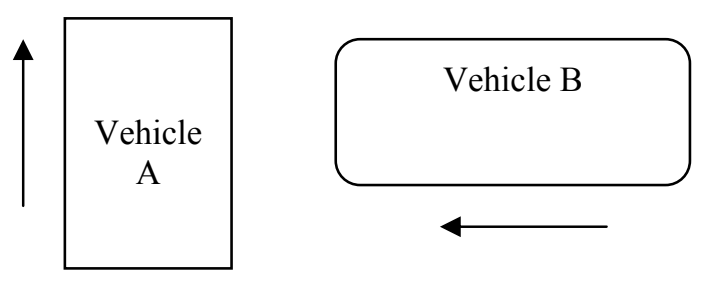

Figure 8

According to consideration 1 the auto brake mechanism of vehicle A will not be activated since the sensor will only detect intensity graph deviation towards the front and rear of vehicle A, based on the front and rear sensor angle, and not towards its sides. So however close vehicle B might come to A, the sensor of A will not activate its auto brake mechanism. Whereas since B is approaching A head on, its sensor would activate the brakes, thus avoiding head on collision for Vehicle B.

\section{B. Sonar Vision Implementation}

In the proposed model, Sonar vision would be implemented so that drivers can avoid backing into objects. The device consists of a sensor mounted on the back of a vehicle about 12 inches from the ground. It is engaged when the vehicle is put into reverse gear. There is a visual and audio warning. Virtually any object that comes within the "Warning Zones" causes the system to beep. The Sonar Vision visual and audio warning component should be installed on the ceiling at the rear of the vehicle cabin. The warning lights are identical in color and significance to traffic lights. This ensures that the driver has quick visual identification of any scenario, at a glance. As stated in Section III, this electronic device will be an added feature that would enhance the safety of the automobile on which it is installed. Along with the USG sensor, the Sonar Vision would avoid head on collision, rear collision and collision during reversing of vehicles.

\section{Steering control through Behavioral Analysis}

This feature would be helpful to steer the vehicle when loss of control would be detected by a VANET node. The proposed model would have a Sensor for monitoring the face of the driver. This Sensor provides input to the Analyzer and the output of the Analyzer determines the state control of the vehicle.

The Analyzer may give the following conclusions over a period of time:

- The driver is feeling sleepy. This could be detected by observing the eyes of the driver. If they remain closed for some period of time then the auto brake mechanism could be activated.

- The driver is feeling uneasy or dizzy. This could be observed by a programmed face recognition system. If the facial expressions of the driver vary drastically from the stored facial images then the brakes would be activated.

Based upon these behavioral analyses the speed and steering could be controlled and auto brake mechanism may be implemented for the safety of the driver and the occupants of the vehicle. Additionally, this feature could be used to identify authenticated drivers to improve the security of the vehicle.

\section{Utility Software}

A utility software would be installed on the VANET node so as to provide additional safety features to the vehicle. The software would be MANET based and would include modules for the following functions:

\section{- GPS based road map generation}

When a vehicle moves, the road map of the adjacent areas would be generated through a GPS based software. This would allow the driver to have an idea of the traffic and he could take an alternate route, if required.

\section{- Minimum distance routing facility}

If multiple routes exist between a source and a destination, the utility software would help the driver to choose the minimum distance path.

\section{- Customized interior adjustments}

The face detection sensor could be utilized to customize the interior adjustments of the vehicle like seat adjustments, rear view mirror angle adjustments etc. This would not only save time for the user but also it will give a pervasive effect to the vehicle.

\section{- Data communication between a VANET node and other mobile devices}

The VANET node will provide for all data communication utilities such as web browser, SMS and MMS facility etc. The MANET routing protocols [6][11] would ensure efficient data packet delivery to the destination. Specialized sensors could be mounted for alerting the emergency services such as ambulance, police and fire brigade.

\section{Limitations of THE Proposed Model}

Although the pervasive automobile model has a lot of safety and additional features, it will not be able to detect a stationary vehicle or any other object (wall, pole, human or cattle). In this model the auto brake mechanism would be activated only in case another vehicle with a VANET node is detected within the front and rear sensor angle limits. This means that VANET nodes should be implemented on all vehicles to avoid head on and rear collisions among vehicles. Thus the major limitations of the proposed model are:

1. The model cannot avoid collisions with stationary objects since it does not implement obstruction detection mechanism.

2. The model requires implementation of VANET nodes on all vehicles that would be moving in a lane or on the roads.

3. Commercial production of all vehicles with VANET nodes is extremely costly.

4. The model requires auto brakes to the activated within milliseconds to avoid collision. Mechanically, this is a serious challenge because of vehicular momentum.

But with the increase in technology, probably the day is not far when safe vehicles would be commercially available for common use.

\section{CONCLUSION}

This paper attempts to project a model of a pervasive vehicle. This model could enhance the security of the automobile occupants and thus reduce the accident mortality rate to a great extent. The major features of the model include:

- Head on and rear collision avoidance based on USG 
and sensor mechanism

- Sonar Vision implementation

- Steering control through behavioral analysis

- GPS based road map generation

- Minimum distance routing facility

- Traffic congestion signaling

- Customized interior adjustments

- Data communication with other mobile devices

- Emergency service alerts

Although this paper has attempted to improve the security features of the automobiles, yet the limitations of the model could be removed in the future work.

\section{ACKNOWLEDGMENT}

We would like to acknowledge the assistance given by our colleagues and institution to carry out this research work. Further we would like to thank the lab co-ordinators of Lakshmi Narain College of Technology and Technocrats Institute of Technology, Bhopal, for all the technical support.

\section{REFERENCES}

[1] http://www.safecarguide.com/exp/statistics/ statistics.htm

[2] http://garage-toys.com/sonvisulsonb.html.

[3] Luis E. Palafox and J. Antonio Garc'1a-Macias. "Wireless Sensor Networks for Voice Capture in Ubiquitous Home Environments". In Proceedings of IEEE International Symposium on Wireless and Pervasive Computing, 2008.

[4] I. Akyildiz,W. Su, Y. Sankarasubramaniam, and E. Cayirci. "A survey on sensor networks". IEEE Communications Magazine, 40(8): 102-114, 2002

[5] W. K. Edwards and R. E. Grinter. "At home with ubiquitous computing: Seven challenges". In Proceedings of the $3 \mathrm{rd}$ international Conference on Ubiquitous Computing, pages 256-272, 2001.

[6] Necla Bandirmali, Ismail Erturk, Celal Ceken. "Securing Data Transfer in Delay-sensitive and Energy-aware WSNs Using. The Scalable Encryption Algorithm". In Proceedings of IEEE International Symposium on Wireless and Pervasive Computing, 2008.

[7] Ricardo Lent and Edith C.-H. Ngai. "Lightweight Clustering in Wireless Sensor-Actuator Networks on Obstructed Environments". In Proceedings of IEEE International Symposium on Wireless and Pervasive Computing, 2008.

[8] Weiser,M. "The Computer for the $21^{\text {st }}$ Century". IEEE Pervasive Computing, Jan-March 2002, pp 19-25.

[9] Vandana Dhingra and Tanvi Rustagi. "Towards Pervasive Computing". In Proceedings of Mobile and Pervasive Computing (CoMPC-2008), pp. 93-96.

[10] Satyanarayanan,M. "Pervasive Computing: Vision and Challenges" IEEE Personal Communication, August 2001, pp. 10-17.

[11] J. Broch, D. A.Maltz, D.B. Johnson, Y.C. Hu, and J.J. etcheva, “ A performance comparison of multi-hop wireless ad hoc network routing protocols". In Proceedings of ACM/IEEE MOBICOM'98, pp.85-97, 1998.

[12] Raya and J.-P. Hubaux. "The security of vehicular ad hoc networks". In Proceedings of SASN'05,2005, pp. 11-21.

[13] M. Raya, A. Aziz and J.-P. Hubaux, "Efficient secure aggregation in VANETs". In Proceedings of VANET'06, 2006. 